The following article has been submitted to Journal of the Acoustical Society of America

\title{
Acoustic Landmarks Contain More Information About the Phone String than Other Frames for Automatic Speech Recognition with Deep Neural Network Acoustic Model
}

\author{
Di He ${ }^{1}$ B Boon Pang Lim $^{2}$, Xuesong Yang ${ }^{3}$, Mark Hasegawa-Johnson ${ }^{3}$, and Deming Chen ${ }^{1}$ \\ ${ }^{1}$ Coordinated Science Lab, University of Illinois at \\ Urbana-Champaign, Urbana, Illinois, USA 61801 \\ ${ }^{2}$ Novumind, Santa Clara, CA 95054 and \\ ${ }^{3}$ Beckman Institute, University of Illinois at \\ Urbana-Champaign, Urbana, Illinois, USA 61801
}

(Dated: March 23, 2018)

\begin{abstract}
Most mainstream Automatic Speech Recognition (ASR) systems consider all feature frames equally important. However, acoustic landmark theory is based on a contradictory idea, that some frames are more important than others. Acoustic landmark theory exploits quantal non-linearities in the articulatory-acoustic and acoustic-perceptual relations to define landmark times at which the speech spectrum abruptly changes or reaches an extremum; frames overlapping landmarks have been demonstrated to be sufficient for speech perception. In this work, we conduct experiments on the TIMIT corpus, with both GMM and DNN based ASR systems and find that frames containing landmarks are more informative for ASR than others. We find that altering the level of emphasis on landmarks by re-weighting acoustic likelihood tends to reduce the phone error rate (PER). Furthermore, by leveraging the landmark as a heuristic, one of our hybrid DNN frame dropping strategies maintained a PER within $0.44 \%$ of optimal when scoring less than half ( $45.8 \%$ to be precise) of the frames. This hybrid strategy out-performs other non-heuristic-based methods and demonstrate the potential of landmarks for reducing computation.
\end{abstract}

Keywords: Automatic Speech Recognition; Acoustic Landmarks; Distinctive Features

*dihe2@illinois.edu 


\section{INTRODUCTION}

Ideas from speech science - which may have the potential to further improve modern automatic speech recognition (ASR) - are not often applied to them (Xiong et al., 2016). Speech science has demonstrated that perceptual sensitivity to acoustic events is not uniform in either time or frequency. Most modern ASR uses a non-uniform frequency scale based on perceptual models such as critical band theory (Fletcher and Munson, 1933). In the time domain, however, most ASR systems use a uniform or frame synchronous time scale: systems extract and analyze feature vectors at regular time intervals, thereby implementing a model according to which the content of every frame is equally important.

Acoustic Landmark Theory (Stevens, 1985, 2000) is a model of experimental results from speech science. It exploits quantal nonlinearities in articulatory-acoustic and acousticperceptual relations to define instances in time (landmarks) at which abrupt changes or local extrema occur in speech articulation, in the speech spectrum, or in a speech perceptual response. Landmark theory proposes that humans perceive phonemes in response to acoustic cues, and that such cues are anchored temporally at landmarks, i.e., that a spectrotemporal pattern is perceived as the cue for a distinctive feature only if it occurs with a particular timing relative to a particular type of landmark. Altering distinctive features alters the phone string; distinctive features in turn get signaled by different sets of cues anchored at landmarks.

The theory of acoustic landmarks has inspired a large number of ASR systems. Acoustic landmarks have been modeled explicitly in ASR system such as those reported in HasegawaJohnson et al., Jansen and Niyogi, Juneja. Many of these systems have accuracies comparable to other contemporaneous systems - in some cases, even returning better performance Hasegawa-Johnson et al. (2005). However, published landmark-based ASR with accuracy comparable to the state of the art has higher computation than the state of the art; conversely, landmark-based systems with lower computational complexity tend to also have accuracy lower than the state of the art. No implementation of acoustic landmarks has yet been demonstrated to achieve accuracy equal to the state of the art at significantly reduced computational complexity. If acoustic landmarks contain more information about the phone string than other frames, however, then it should be possible to significantly reduce computational complexity of a state of the art ASR without significantly reducing accuracy, 
or conversely, to increase accuracy without increasing computation, by forcing the ASR to extract more information from frames containing landmarks than from other frames.

We assume that a well trained frame-synchronous statistical acoustic model (AM), having been trained to represent the association between MFCC features and triphones, has also learned sufficient cues and necessary contexts to associate MFCCs and distinctive features. However, because the AM is frame-synchronous, it must integrate information from both informative and uninformative frames, even if the uninformative frames provide no gain in accuracy. The experiments described in this paper explore whether, if we treat frames containing acoustic landmarks as more important than other frames, we can get better accuracy or lower computation. In this work, we present two methods to quantify the information content of acoustic landmarks in an ASR feature string. In both cases, we use human annotated phone boundaries to label the location of landmarks. The first method seeks to improve ASR accuracy by over-weighting the AM likelihood scores of frames containing phonetic landmarks. By over-weight, we mean multiplying log-likelihoods with a value larger than 1 (Section III A). The second method seeks to reduce computation, without sacrificing accuracy, by removing frames from the ASR input. Removing frames makes the computational load decrease, but usually causes accuracy to decrease also; which frames can be removed that cause the accuracy to drop the least? We searched for a strategy that removes as many frames as possible while attempting to keep the Phone Error Rate (PER) low. We show that if we know the locations of acoustic landmarks, and if we retain these frames while dropping others, it is possible to reduce computation for ASR systems with a very small error increment penalty. This method for testing the information content of acoustic landmarks is based on past works (Iso-Sipilä, 2000, McGraw et al., 2016, Vanhoucke et al., 2013) that demonstrated significantly reduced computation by dropping acoustic frames, with small increases in PER depending on the strategy used to drop frames. In this paper we adopt the PER increment as an indirect measure of the phonetic information content of the dropped frames.

If the computational complexity of ASR can be reduced without sacrificing accuracy, or if the accuracy can be increased without sacrificing computation, these findings should have practical applications. It is worth emphasizing that this work only intends to explore these potential applications, assuming landmarks can be accurately detected. Our actual acoustic landmark detection accuracy, despite increasing over time, has not reached a practical level 
yet.

In this paper, Section II briefly reviews the acoustic landmark theory and relevant works which apply it to ASR systems. Section III presents the theoretical basis for our experiments. Section[V]proposes the hypothesis. Experimental setup is explained in Section V and results are presented in SectionVI. Discussion, including a case study of the confusion characteristics is presented in Section VII. At last, our conclusions are drawn in Section VIII.

\section{BACKGROUND AND LITERATURE REVIEW}

Acoustic landmark theory was first proposed as a theory of the perception of distinctive features, therefore many landmark-based ASRs use distinctive features rather than triphones (Lee, 1988) as their finest-grain categorical representation. Distinctive features are an approximately binary encoding of perceptual (Jakobson et al., 1951), phonological (Chomsky and Halle, 1968), and articulatory (Stevens, 1985) speech sound categories. A feature is called "distinctive" if and only if it defines a phoneme category boundary, therefore distinctive features are language dependent. The distinctive features used by each language often have articulatory, acoustic, and/or perceptual correlates that are similar to those of distinctive features in other languages, however Stevens, 2002, Stevens et al. 1986), so it is possible to define a set of approximately language-independent distinctive features as follows: if an acoustic or articulatory feature is used to distinguish phonemes in at least one of the languages of the world, then that feature may be considered to define a language-independent distinctive feature. Each phoneme of a language is a unique vector of language-dependent distinctive features. Automatic speech recognition may distinguish two different allophones of the same phoneme as distinct phones; in most cases, the distinctions among phones can be coded using distinctive features borrowed from another language, or equivalently, from the language-independent set.

The ASR community has explored a number of encodings similar to distinctive features, e.g., articulatory features (Kirchhoff, 1998, 1999, Kirchhoff et al., 2002, Livescu et al., 2007, Metze, 2005, Naess et al., 2011) and speech attributes (Lee et al., 2007). These concepts have different foci, but are also very similar. Distinctive features are defined by phoneme distinctions, therefore they are language dependent. It is possible to define a languageindependent set of distinctive features based on quantal nonlinearities in the articulatory- 
acoustic Stevens (1972) and acoustic-perceptual Stevens (1985) transformations. Although both the articulatory-acoustic and acoustic-perceptual transformations contain quantal nonlinearities that may define distinctive features, a much larger number of nonlinearities in the articulatory-acoustic transformation has been demonstrated. Many studies therefore focus only on the set of phoneme distinctions defined by nonlinearities in the articulatory-acoustic transformation, which are called "articulatory features" in order to denote their defining principle. Of these, some studies focus on the articulatory-acoustic transform because it implies a degree of acoustic noise robustness (Kirchhoff, 1998, 1999, Kirchhoff et al., 2002), others because it implies a compact representation of pronunciation variability Livescu et al. (2007), others because it is demonstrably language-independent Metze (2005), Naess et al. (2011). Speech attributes, on the other hand, are a super-set of distinctive features; they are deliberately defined to introduce other purposes to speech recognition. In Lee's framework (Lee et al. 2007), speech attributes are quite broadly defined to be perceptible speech categories, of which phonological categories are only a subset. Under this definition, speech attributes include not only distinctive feature but also a wide variety of acoustic cues signaling gender, accent, emotional state and other prosodic, meta-linguistic, and para-linguistic messages.

As opposed to modern statistical ASR where each frame is treated with equal importance, landmark theory proposes that there exist information rich regions in the speech utterance, and that we should focus on these regions more carefully. These regions of interest are anchored at acoustic landmarks. Landmarks are instantaneous speech events near which distinctive features are most clearly signaled. These key points mark human perceptual foci and key articulatory events (Liu, 1996). Stevens (1985) first introduced these instantaneous speech events, where, for some phonetic contrasts, humans focus their attention in order to extract acoustic cues necessary for identifying the underlying distinctive features. Initially Stevens named these key points "acoustic boundaries"; the name "acoustic landmarks" was introduced in 1992 (Stevens et al. $)$, and has been used consistently since. At roughly the same time Furui (1986) and Ohde (1994) made similar observations when studying children's speech perception in Japanese.

(Liu, 1996) demonstrated algorithms for automatically detecting acoustic landmarks. (Hasegawa-Johnson, 2000) measured the phonetic information content of known acoustic landmarks. In his work, Hasegawa-Johnson (2000) defined a set of landmarks including 
consonant releases and closures (at phone boundaries) and vowel/glide pivot landmarks (near the center of the corresponding phones). In contrast, Lulich (2010) argued that the center of vowels and glides are not as informative and should not be considered as landmarks. He defined, instead, formant-subglottal resonance crossing, which is known to sit between boundaries of [-back] and [+back] vowels, to be more informative. In a paper, Wang et al. (2009) showed that the latter improves performance for automatic speaker normalization application. In (Hasegawa-Johnson, 2000), a small number of pivot and release landmarks were defined at $+33 \%$ and $-20 \%$ locations after the beginning or before the end of certain phones (where $+33 \%$ indicates delaying the location by $33 \%$ of the total duration of that phone; $-20 \%$ indicates advancing the location by $20 \%$ ), in order to better approximate the typical timing of the spectrotemporal events defined earlier in Liu's work (Liu, 1996). Later works (Hasegawa-Johnson et al., 2005, Kong et al., 2016) labeled these landmarks right on the boundary and returned similar performance with Hasegawa-Johnson (2000). Figure 1 illustrates the landmark labels for the pronunciation of word "Symposium" " The details landmark labeling heuristics applied in this example are further described in Table \&.

\footnotetext{
${ }^{1}$ The pronunciation of "Symposium" is selected from audio file: TIMIT/TRAIN/DR1/FSMA0/SX361.WAV
} 


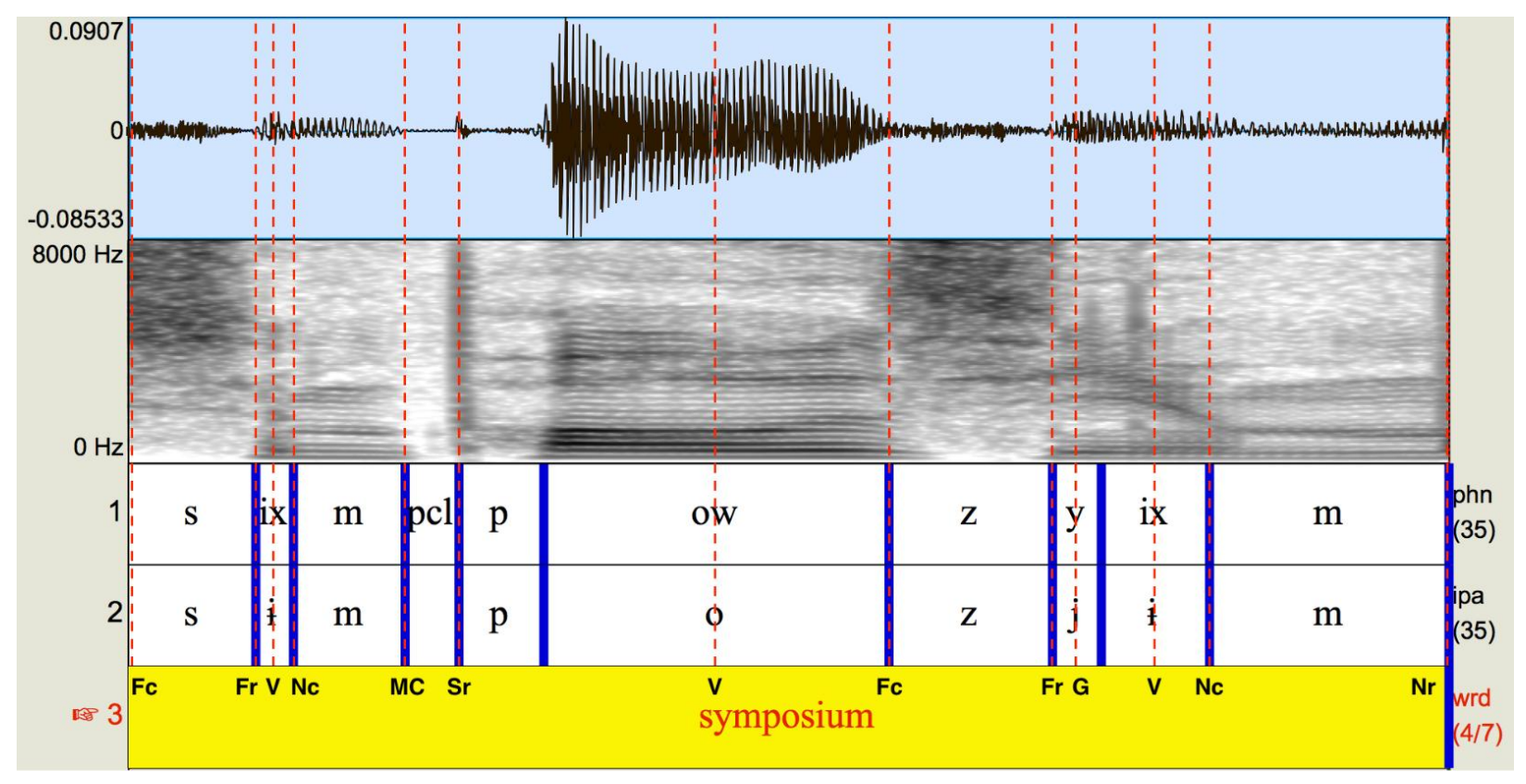

FIG. 1: Acoustic landmark labels (LM) for the pronunciation of the word "Symposium". TIMIT phone symbols (PHN) and international phonetic alphabet (IPA) symbols are both used in this example. The dashed red lines denote the landmark positions. The symbols under the dashed red lines are landmark types, where $\mathbf{F c}$ and $\mathbf{F r}$ are closure and release for fricatives; $\mathbf{S c}$ and $\mathbf{S r}$ are closure and release for Stops; $\mathbf{N c}$ and $\mathbf{N r}$ are closure and release for nasals; $\mathbf{V}$ and $\mathbf{G}$ are vowel pivot and glide pivot; $\mathbf{M C}$ is manner-change landmark.

Many works have focused on accurately detecting acoustic landmarks. The first of these assumed that landmarks correspond to the temporal extrema of energy or energy change in particular frequency bands, e.g., Liu (1996) detected consonantal landmarks in this way, Howitt (2000) detected vowel landmarks, Choi (1999) classified consonant voicing, and Lee and Choi (2008a), Lee et al. (2011, 2012), Lee and Choi (2008b) classified place of articulation. Support Vector Machines (SVMs) were popularized for landmark detection by Niyogi, Burges, and Ramesh (1999), who showed that an SVM trained to observe a very small acoustic feature vector (only four measurements, computed once per millisecond) can detect stop release landmarks more accurately than a hidden Markov model. Both Borys (2008) and Chitturi and Hasegawa-Johnson (2006) target the detection of all landmarks using one kind of acoustic features. Their results are reasonably accurate, but are still less accurate and more computationally expensive than the best available classifier for each distinctive feature. Xie and Niyogi (2006) expanded the work of Niyogi, Burges, and Ramesh (1999) by demonstrating detection of several different types of landmark using a very small acoustic feature vector. In Qian's paper (Qian et al., 2016), a small vector of acoustic fea- 
tures was learned, using the technique of local binary patterns, and resulting in accuracy above $95 \%$ for stop consonant detection. In a paper from Kong et al. (2016), a Convolutional Neural Network (CNN) trained on MFCC and additional acoustic features achieved around $85 \%$ on consonant voicing detection. This system was trained on the English corpus TIMIT (Garofalo et al., 1993), but tested on Spanish and Turkish corpora. Over time, new techniques and more specific features have been developed for landmark detection, and the detection accuracy has been improving steadily. Acoustic landmarks were first introduced as part of an ASR in 1992 (Stevens et al.), and have been used in a variety of ASR system architectures. These systems, without considering the mechanism used for landmark detection, can be clustered into two types. The first type of system, such as those described by Jansen and Niyogi (2008), Juneja (2004), Liu (1996) computes a lexical transcription directly from a set of detected distinctive features. Due to the complexity of building a full decoding mechanism on distinctive features, some of these systems only output isolated words. However, other systems (e.g.,work from Jansen and Niyogi (2008)) have full HMM back-ends that can output word sequences. The other type of system, such as that described by Hasegawa-Johnson et al. (2005), conducts landmark-based re-scoring on the lattices generated by an MFCC-based hidden Markov model. Acoustic likelihoods from the classic ASR systems are adjusted by the output of the distinctive feature classifier. Many landmark based ASRs demonstrated performance slightly (Hasegawa-Johnson et al., 2005) or even significantly (Kirchhoff, 1998) better than baseline ASR systems, especially in noisy conditions.

\section{MEASURES OF THE INFORMATION CONTENT OF ACOUSTIC FRAMES}

An acoustic landmark is an instantaneous event that serves as a reference time point for the measurement of spectrotemporal cues widely separated in time and frequency. For example, in the paper that first defined landmarks, Stevens proposed classifying distinctive features of the landmark based on the onsets and offsets of formants and other spectrotemporal cues up to $50 \mathrm{~ms}$ before or $150 \mathrm{~ms}$ after the landmark (Stevens, 1985). The $200 \mathrm{~ms}$ spectrotemporal dynamic context proposed by Stevens is comparable to the $165 \mathrm{~ms}$ spec-

trotemporal dynamic context computed for every frame by the ASR system of Veselý et al. (2013). Most ASR systems use acoustic features that are derived from frames 25ms long, 
with a 10ms skip, as human speech is quasi-stationary for this short period (Quatieri, 2008). Because spectral dynamics communicate distinctive features, however, ASR systems since 1981 (Furui) have used dynamic features; since deep neural nets (DNNs) began gaining popularity, the complexity of the dynamic feature set in each frame has increased quite a lot, with consequent improvements in ASR accuracy. This trend not only applies to stacking below 100ms. With careful normalization, features like TRAPs (Hermansky, 2003), with temporal window equal or longer than $500 \mathrm{~ms}$, continue to demonstrate accuracy improvement. Experiments reported in this paper are built on a baseline described by Veselý et al. (2013), and schematized in Fig. 2. In this system, MFCCs are computed once every 10ms, with $25 \mathrm{~ms}$ windows (dark gray rectangles in Fig 2). In order to include more temporal context, we stack adjacent frames, three preceding and three succeeding, for a total of seven frames (a total temporal spanof $(7-1) \times 10+25=85 \mathrm{~ms}$ ). These are shown in Fig 2 as the longer, segmented dark gray rectangles, with the red segments representing the center frames of each stack. The seven-frame stack is projected down to 40 dimensions using linear discriminant analysis (LDA). For input to the DNN but not the GMM, LDA is followed by speaker adaptation using mean subtraction and feature-space maximum likelihood linear regression, additional context is provided by a second stacking operation afterwards, in which LDA-transformed features, represented by yellow rectangles, are included in stacks of 9 frames (for a total temporal span of $(9-1) \times 10+85=165 \mathrm{~ms}$ ), as represented by the top path in Fig 2, It is believed that the reason features spanning longer duration improve ASR accuracy is that long lasting features capture coarticulation better, including both neighboring-phone transitions and longer-term coarticulation. The dynamics of the tongue naturally cause the articulation of one phoneme to be reflected in the transitions into and out of neighboring phonemes, over a time span of perhaps 70ms. Longer-term coarticulation, spanning one or more syllables, can occur when an intervening phoneme does not require any particular placement of one or more articulators, e.g., Öhman (1965) demonstrated that the tongue body may transition smoothly from one vowel to the next without apparently being constrained by the presence of several intervening consonants. 


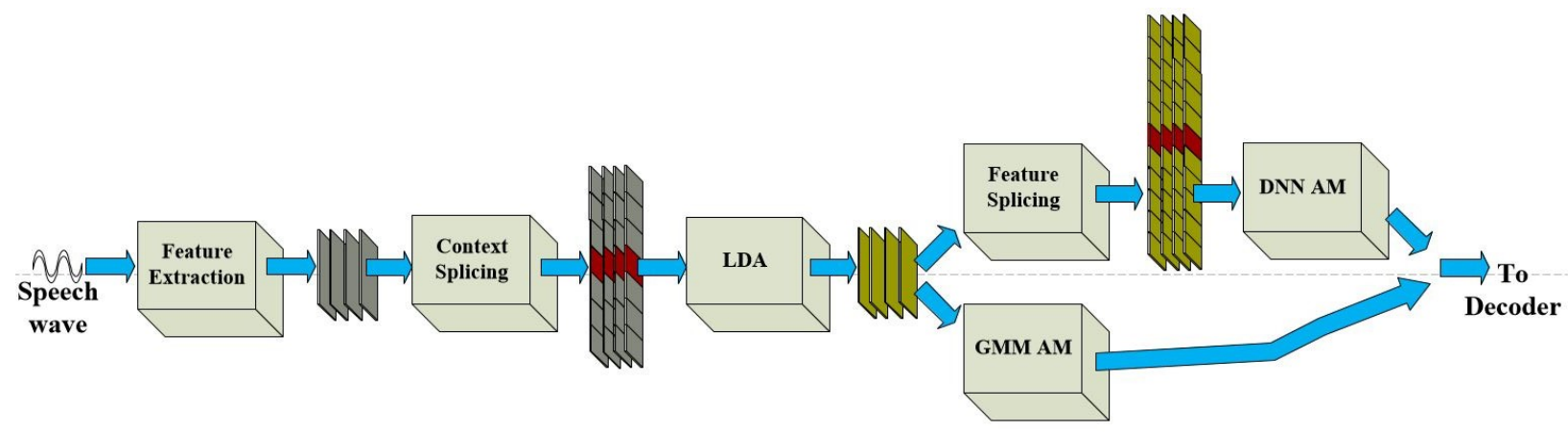

FIG. 2: Stacking of Feature Frames Before the Scoring Process for DNN AM (top path) and GMM AM (bottom path). The dark gray, red and green rectangles indicate frames and stacks of frames. Please see text.

\section{A. Frame Re-weighting}

HMM-based ASR searches the space of all possible state sequences for the most likely state sequence given the observations. During the state likelihood estimation, results of all frames are weighted equally. Weighting more informative frames more heavily could potentially benefit speech recognition. Ignoring the effects of the language model, the log-likelihood of a state sequence $S$ given the observations $O$ is

$$
L(S \mid O)=\sum_{t=1}^{T} w(t) \log \left(p\left(o_{t} \mid s_{t}\right)\right)+\log \left(p\left(s_{t} \mid s_{t-1}\right)\right)
$$

where $s_{t}$ and $o_{t}$ are respectively the state and observed feature vector associated with the frame at time $t$. The state $s_{t}$ at any time should be associated with one of the senones (i.e., monophone or clustered triphone states). Here $p\left(s_{t} \mid s_{t-1}\right)$ is the transition probability between senones, which we will not consider modifying in this study. In most systems, beam search parameters constrain the number of active states, thus we only need to evaluate the necessary posteriors. In our over-weighting framework, if $o_{t}$ contains a landmark, the value of $\log p\left(o_{t} \mid s_{t}\right)$ will be scaled. To simplify the computation, we operate directly on loglikelihoods. In this case, $\log \left(p\left(o_{t} \mid s_{t}\right)\right)$ is multiplied by factor $w(t)$ which takes the value 1 when frame $t$ contains no landmark and a value greater than 1 otherwise. This is effectively applying a power operation on the likelihoods.

The key in this strategy is that the likelihood of all model states will be re-weighted. If the frame over-weighted is a frame that can differentiate the correct state better, the error 
rate will drop. In contrast, if the likelihood of a frame is divided evenly across states, or even worse, is higher for the incorrect state, then over-weighting this frame will mislead the decoder and increase chances of error. For this reason, over-weighting landmark frames is a good measure to tell how meaningful landmark frames are compared to the rest of the frames. If the landmarks are indeed more significant, we should observe a reduction in the PER for the system over-weighting the landmark.

\section{B. Frame dropping}

The wide temporal windows used in modern ASR, as mentioned in the beginning of Section III, are highly useful to landmark-based speech recognition: all of the dynamic spectral cues proposed by Stevens 1985 are within the temporal window spanned by the feature vector of a frame centered at the landmark, therefore it may be possible to correctly identify the distinctive features of the landmark by dropping all other frames, and keeping only the frame centered at the landmark. Our different frame dropping heuristics modify the $\log$ probability of a state sequence by replacing the likelihood $p\left(o_{t} \mid s_{t}\right)$ with an approximation function $f$. In terms of log probabilities, Equation (1) becomes

$$
L(S \mid O)=\sum_{t=1}^{T} \log f\left(p\left(o_{t} \mid s_{t}\right), t\right)+\log \left(p\left(s_{t}, s_{t-1}\right)\right),
$$

The class of optimizations considered in this paper involve a set of functions $f\left(p\left(o_{t} \mid s_{t}\right)\right)$ parameterized as:

$$
f\left(p\left(o_{t} \mid s_{t}\right)\right)=\left\{\begin{array}{ll}
R(O, t) & \text { if } g(t)=1 \\
p\left(o_{t} \mid s_{t}\right) & \text { otherwise }
\end{array},\right.
$$

The method of replacement is characterized by $R$, and the frame-dropping function by $g(t)$. This work considers multiple methods to verify that the finding with respect to landmarks is independent of the replacement method. The four possible settings of the $R(o, t)$ function 
are as follows:

$$
R(O, t) \in\left\{\begin{array}{l}
R_{\text {Copy }}(O, t)=p\left(o_{t^{\prime}} \mid s_{t^{\prime}}\right), \quad t^{\prime}=\max _{\tau \leq t, g(\tau)=0} \tau \\
R_{\text {Fill_o }}(O, t)=1 \\
R_{\text {Fill_const }}(O, t)=\left(\prod_{t=1}^{T} p\left(o_{t} \mid s_{t}\right)\right)^{1 / T} \\
R_{\text {Upsample }}(O, t)=\exp \left(\sum_{t^{\prime}: g\left(t^{\prime}\right)=0} h\left(t-t^{\prime}\right) \log p\left(o_{t} \mid s_{t}\right)\right)
\end{array}\right.
$$

In other words, the Copy strategy copies the most recent observed value of $p\left(o_{t} \mid s_{t}\right)$, the Fill_0 strategy replaces the log probability by 0 , the Fill_const strategy replaces the log probability by its mean value, and the Upsample strategy replaces it by an interpolated value computed by interpolating (using interpolation filter $h(t)$ ) the log probabilities that have been selected for retention. The Upsample strategy will only be used if the frame-dropping function is periodic, i.e., if frames are downsampled by a uniform downsampling rate.

The pattern of dropped frames can be captured by the indicator function $g$, which is true for frames that we want to drop. Experiments will test two landmark-based patterns: Landmark-drop drops all landmark frames $(g(t)=1$ if the frame contains a landmark), and Landmark-keep keeps all landmark frames $(g(t)=1$ only if the frame does not contain a landmark). In the case where landmark information is not available, the frame-dropping pattern may be Regular, in which $g(t)=\delta(t \bmod K)$ indicating that every $K$-th frame is to be dropped, or it may be Random, in which case the indicator function is effectively a binary random variable set at a desired frame dropping rate. As we will demonstrate later, to achieve a specific function and dropping ratio, we can sometimes combine output of different $g$ functions together by taking a logical inclusive OR to their output.

If acoustic landmark frames contain more valuable information than other frames, it can be expected that experiment setups that retain the landmark frames should out-perform

other patterns, while those that drop the landmark frames should under-perform, regardless of the method of replacement chosen.

\section{HYPOTHESES}

This paper tests two hypotheses. The first is that a window of speech frames (in this case 9 frames) centered at a phonetic landmark has more information than windows centered 
elsewhere - this implies that over-weighting the landmark-centered windows can result in a reduction in PER. The second hypothesis states that keeping landmark-centered windows rather than other windows causes little PER increment, and that dropping a landmarkcentered window causes greater PER increment as opposed to dropping other frames. In the study we focused on PER as opposed to Word Error Rate (WER) for two reasons. First, the baseline Kaldi recipe for TIMIT reports PER. Second, this study is oriented towards speech acoustics; focusing on phones allow us to categorize and discuss the experiment and results in better context.

TABLE I: Landmark types and their positions for acoustic segments. $\boldsymbol{F c}$ and $\boldsymbol{F r}$ are closure and release for fricatives; $\boldsymbol{S c}$ and $\boldsymbol{S r}$ are closure and release for Stops; $\boldsymbol{N} \boldsymbol{c}$ and $\boldsymbol{N} \boldsymbol{r}$ are closure and release for nasals; $\boldsymbol{V}$ and $\boldsymbol{G}$ are vowel pivot and glide pivot; 'start', 'middle', and 'end' denote three positions across acoustic segments.

\begin{tabular}{||l|l|l||}
\hline $\begin{array}{l}\text { Manner of } \\
\text { Articulation }\end{array}$ & $\begin{array}{l}\text { Landmark Type and } \\
\text { Position }\end{array}$ & Observation in Spectrogram \\
\hline Vowel & V: middle & maximum in low- and mid-frequency amplitude \\
Glide & G: middle & minimum in low- and mid-frequency amplitude \\
\hline Fricative & Fc: start, Fr: end & \\
Affricate & Sr,Fc: start, Fr: end & amplitude discontinuity occurs when consonantal \\
Nasal & Nc: start, Nr: end & constriction is formed or released \\
Stop & Sc: start, Sr: end & \\
\hline
\end{tabular}

In order to test these hypotheses, a phone boundary list from the TIMIT speech corpus (Garofalo et al., 1993 was obtained, and the landmarks were labeled based on the phone boundary information. Table I briefly illustrates the types of landmarks and their positions, as defined by the TIMIT phone segments. This marking procedure is shared by Hasegawa-Johnson et al. (2005), Kong et al. (2016), Stevens (2002). It is worth mentioning that this definition disagrees with that of Lulich (2010). Lulich claims that there is no landmark in the center of Vowel and Glide; instead, a formant-subglottal resonance crossing, which is known to sit between the boundaries of [-Back] and [+Back] vowels, contains a landmark. Frames marked as landmark are of interest. To test hypothesis 1, landmark frames are over-weighted. To test hypothesis 2, either non-landmark or landmark frames are dropped. 


\section{EXPERIMENTAL METHODS}

Our experiments are performed on the TIMIT corpus. Baseline systems use standard examples distributed with the Kaldi open source ASR toolkit2. Specifically, the GMM-based baseline follows the configurations in the distributed tri2 configuration in the Kaldi TIMIT example files 3 . The clustered triphone models are trained using maximum likelihood estimation of features that have been transformed using linear discriminant analysis and maximum likelihood linear transformation. For the DNN baseline, speaker adaptation is performed on the features, and nine consecutive frames centered at the current frame are stacked as inputs to the DNN, as specified in the distributed tri4_nnet example. Respectively, the two systems achieved PER of 23.8\% (GMM) and 22.6\% (DNN) without any modification.

We performed a 10-fold cross validation (CV) over the full corpus, by first combining the training and test sets, and creating 10 disparate partitions for each test condition. The gender balance was preserved to be identical to the canonical test set for each test subset, while the phonetic balance was approximately the same but not necessarily identical. This is in order to improve the significance of our PER numbers. The TIMIT corpus is fairly small and the phone occurrence of some phones, or even phone categories, in the test set is lower than ideal. Conducting cross validation on the full set allows us partially address this issue.

For the control experiments of our tests, all configurations of feature extraction and decoding process are retained the same as the baseline. In this case, fair comparisons are guaranteed, and we can fully reveal the effects of our methods in the AM scoring process.

\section{EXPERIMENTAL RESULTS}

Experimental results examining the two hypotheses proposed above will be presented in this section. We will present the results of over-weighting the landmark frames first. Evaluation of frame dropping will be presented second, and includes several phases. In the first phase, a comparison of different methods of replacement is presented, to provide the reader with more insight into these methods before they are applied to acoustic landmarks. In the second phase, we will then leverage our findings to build a strategy that both drops

\footnotetext{
2 http://kaldi-asr.org/
}

3 hhttps://github.com/kaldi-asr/kaldi/tree/master/egs/timit/s5 
non-landmark frames, and over-weights landmark frames, using the best available pattern of dropped frames and method of replacement.

\section{A. Hypothesis 1: Over-weighting Landmark Frames}

Figure 3 illustrates the PER of the strategy of over-weighting the landmark frames during the decoding procedure, and how it varies with the factor used to weight the AM likelihood of frames centered at a landmark. The PER for GMM-based models drops as the weighting factor increases until the factor is 1.5; increasing the weighting factor above 1.5 causes the PER to increase slightly. When the factor is increased to greater than 2.5, the PER increases at a higher slope. Similar trends can be found for DNN models, yet in this case the change in PER is non-concave and spans a smaller range. If landmark frames are under-weighted, or over-weighted by a factor of 1.5 or up to 2.0, PER increases. Over-weighting landmark frames by a factor of 3.0 to 4.0 reduces PER. In this experiment, Wilcoxon tests Gillick and Cox, 1989) have been conducted, through Speech Recognition Scoring Toolkit (SCTK) 2.4.10 4 and tests concluded the difference to be insignificant.

\section{B. Methods of Replacement for Dropped Frames}

Figure 4 compares the performance of three methods of replacement: Copy, Fill_0 and Fill_const when a Regular frame dropping pattern is used. Results show that Fill_0 and Fill_const suffer very similar PER increments as the percentage of frames dropped is increased, while Copy shows a relatively smaller PER increment for drop rates of $40 \%$ or 50\%. As for the comparison between acoustic models, DNN-based models outperform GMM-based at all drop rates. Notably, the Copy approach synergizes well with DNN models, and is able to maintain low PER increments even up to $75 \%$ drop rate; this finding is similar to findings reported in papers from Vanhoucke et al. (2013).

Figure 5 compares the performance between two patterns of dropping frames - Regular, Random. In both of these the Copy method for replacement was used. We also provide for comparison, the Regular pattern, but using an Upsample replacement method. This scheme uses a 17-tap anti-aliasing FIR filter. The method that offered the lowest phone error rate

\footnotetext{
4 https://www.nist.gov/itl/iad/mig/tools
} 

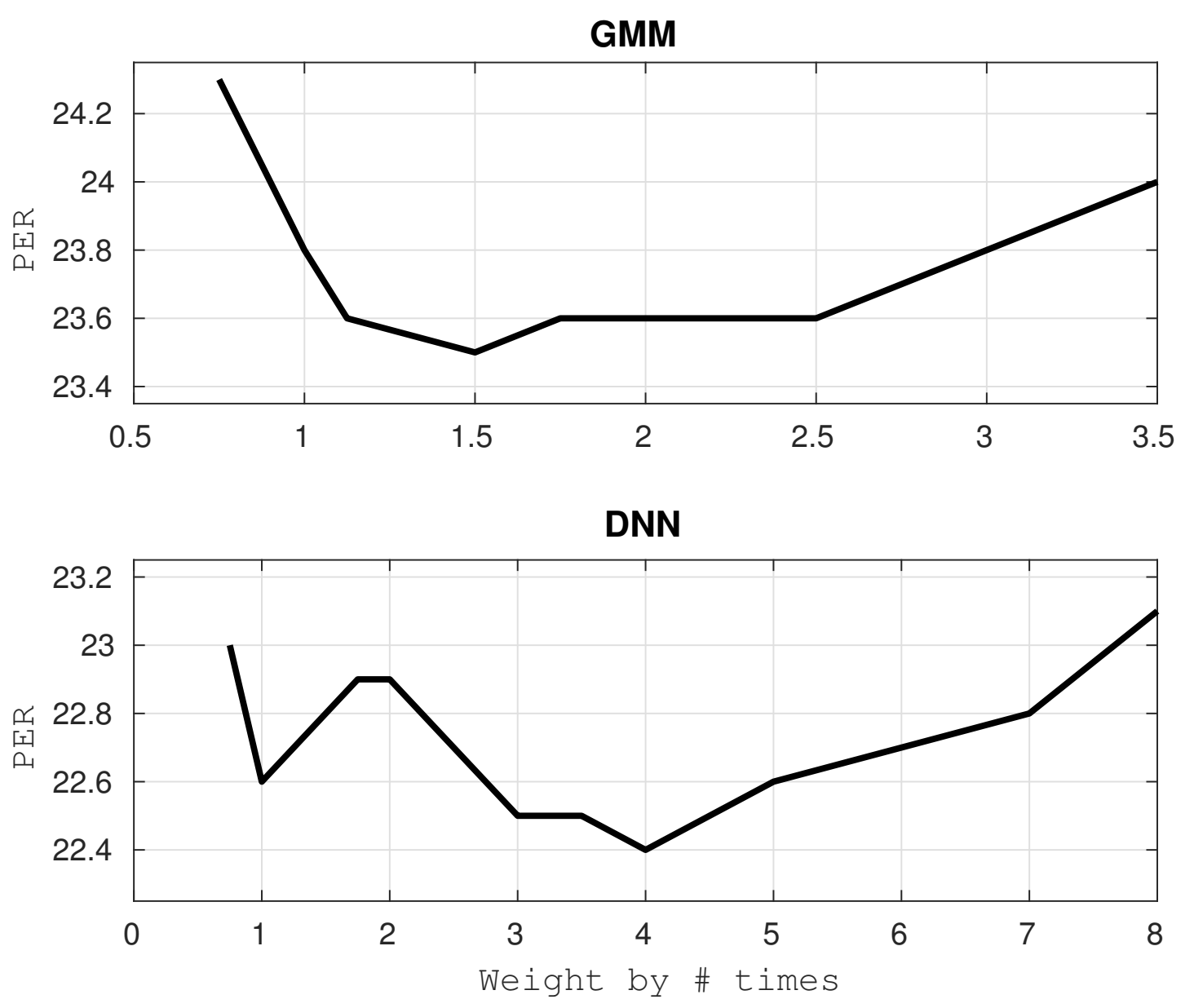

FIG. 3: Over-weighting landmark frames for GMM and DNN.

increment is obtained using a Regular pattern with a Copy replacement scheme. Results show that Regular-Copy outperforms other methods by a large margin in terms of PER increment independent of which AM is used.

\section{Hypothesis 2: Dropping Frames with Regards to Landmarks}

At the beginning of this section, experiments that test hypothesis 2 directly are described. The focus is to subject the ASR decoding process to frames missing acoustic likelihood scores, and see how the decoding error rate changes accordingly. Obviously we are interested in using the presence vs. absence of an acoustic landmark as a heuristic to choose the frames to keep or drop. To quantify the importance of the information kept vs. the information discarded, dropping strategies (Landmark-keep and Landmark-drop) are compared to the 

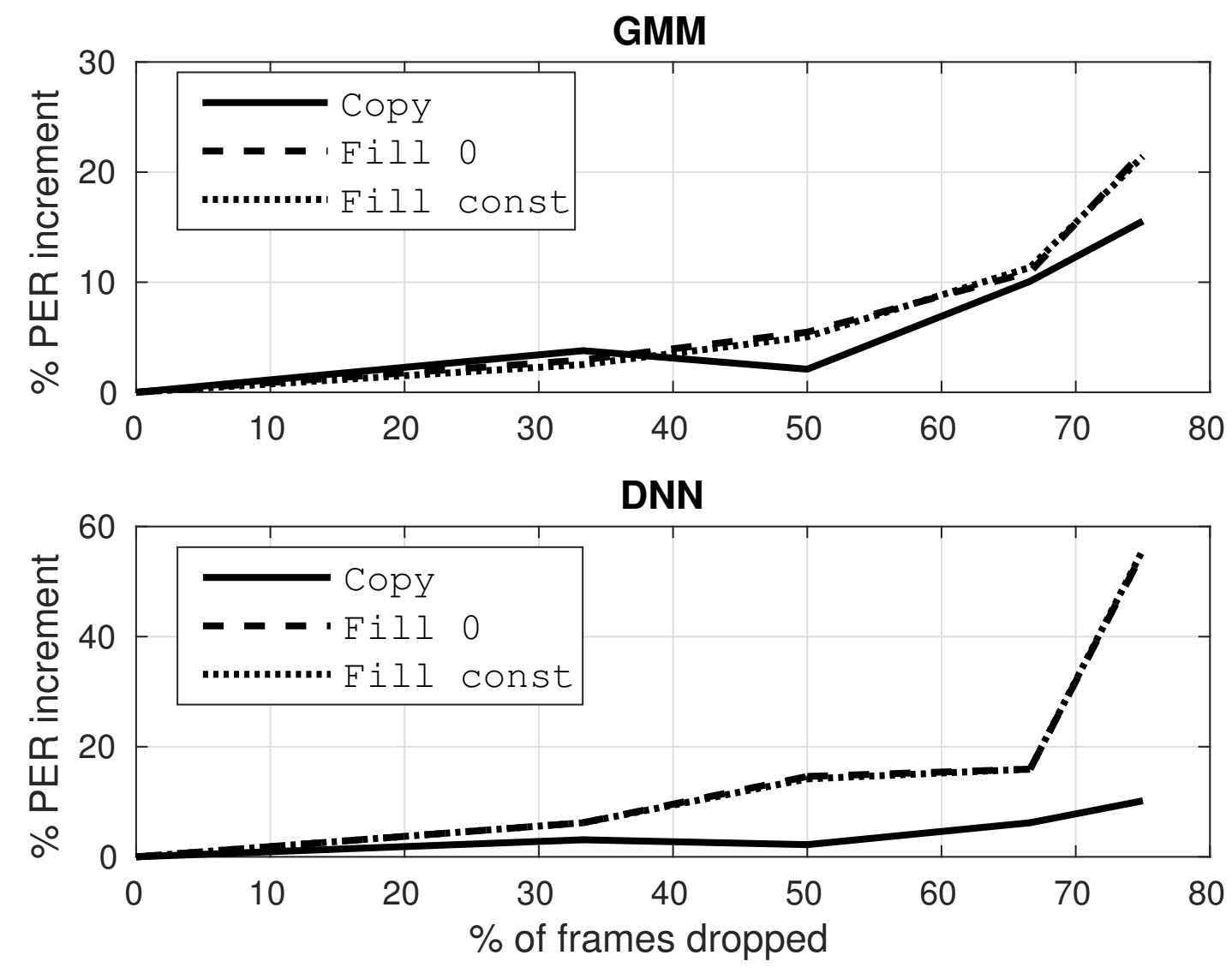

FIG. 4: Comparison of Different Methods of Frame Replacement (Copy, Fill_0 and Fill_const) assuming a Regular pattern of frame replacement.

non-landmark-based Random strategy. Notice the Regular strategy has been shown to be more effective than Random (e.g., in Fig. 5), however, to make the PER result meaningful, the same number of frames should be dropped across different patterns being compared. When we keep only landmarks (Landmark-keep) or drop only landmarks (Landmark-drop), the percentage of frames dropped can not be precisely controlled by the system designer: it is possible to adjust the number of frames retained at each landmark (thus changing the drop rate), but it is not possible to change the number of landmarks in a given speech sample. Therefore, precisely adjusting the drop rate to meet a different pattern is not practical. Depending on the test set selected, the portion of frames containing landmarks ranges from $18.5 \%$ to $20.5 \%$. As opposed to Random, Regular does not give us the ability to select a drop rate that exactly matches the drop rate of the Landmark-drop or Landmark-keep strategies. Therefore, it is not covered in the first 2 experiments. However, in the 3rd experiment, we will compare a frame dropping strategy using landmark as heuristic against Regular 

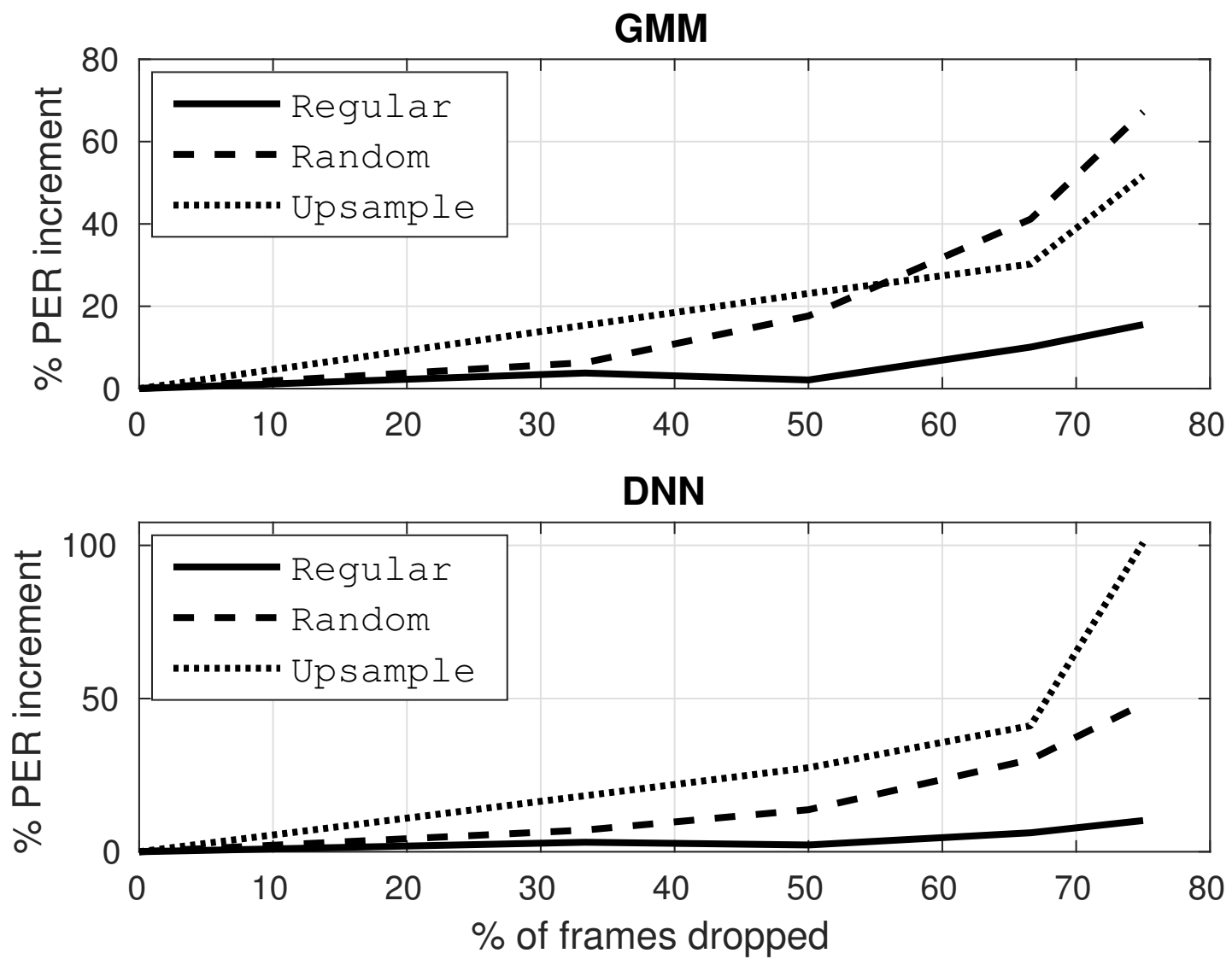

FIG. 5: Comparison of Different Patterns of Dropping Frames assuming Copy (Regular and Random) and Interpolation through low-pass filtering (Upsample) method of replacement.

dropping. But that experiment will serve a slightly different purpose.

As in the over-weighting experiment, two types of frame replacement are tested. The Fill_0 strategy is an exact implementation of hypothesis 2: when frames are dropped, they are replaced by the least informative possible replacement (a log probability of zero). Figure 4 showed, however, that the Copy strategy is more effective in practice than the Fill_0 strategy, therefore these two strategies are tested using a landmark-based frame drop pattern. Figure 4 showed that the Fill_const strategy returns almost identical results to Fill_0, so it is not separately tested here.

Experiment results are presented for both the TIMIT default test split, and for crossvalidation $(\mathrm{CV})$ using the whole corpus. The baseline implementation is as distributed with the Kaldi toolkit. Since no frames are dropped, it returns the lowest PER. However, likelihood scoring for the baseline AM will require more computation when com- 
pared to a system that drops frames. For CV we report the mean relative PER increment $(\triangle \mathrm{PER}=100 \times($ modified PER - baseline PER $) /($ baseline PER $))$, with its standard deviation in parentheses, across all folds of CV. Every matching pair of frame-drop systems (Landmark-keep versus Random) is tested using a two-sample $t$-test (Cressie and Whitford, 1986), across folds of the CV, in order to determine whether the two PER increments differ. During the $t$-test, we assume PER numbers from different folds are samples of a random variable. The two-sample $t$-test intends to find out whether the random variables representing PER for different setups (Landmark-keep versus Random) have the same mean.

\section{Keeping or Dropping the Landmark Frames}

Table II illustrates the changes in PER increment that result from a Landmark-keep strategy (score only landmark frames) versus a Random frame-drop strategy set to retain the same percentage of frames. For each test set, we count the landmark frames separately and match the drop rate exactly between the Landmark-keep and Random strategy. In all cases, the Landmark-keep strategy has a lower PER increment. A Wilcoxon test has been conducted on the default test set; the difference between all pairs but the DNN Fillo pair is significant.

TABLE II: PER increments for scoring Landmark frames only compared to randomly dropping similar portion of frames ( $C V$ stands for cross validation; if the two increments differ, then the lower of the two is marked with either $*(p<0.05)$ or $* *(p<0.001)$.)

\begin{tabular}{|c|c|c|c|c|c|c|c|c|}
\hline Acoustic model & \multicolumn{4}{|c|}{ GMM } & \multicolumn{4}{|c|}{$\mathrm{DNN}$} \\
\hline Test regime & \multicolumn{2}{|c|}{ Default } & \multicolumn{2}{|c|}{ CV Mean (Stdev) } & \multicolumn{2}{|c|}{ Default } & \multicolumn{2}{|c|}{ CV Mean (Stdev) } \\
\hline Metric & $\begin{array}{c}\text { PER } \\
(\%)\end{array}$ & $\begin{array}{c}\text { PER Inc } \\
(\%)\end{array}$ & $\begin{array}{c}\text { PER } \\
(\%)\end{array}$ & $\begin{array}{c}\text { PER Inc } \\
(\%)\end{array}$ & $\begin{array}{c}\text { PER } \\
(\%)\end{array}$ & $\begin{array}{c}\text { PER Inc } \\
(\%)\end{array}$ & $\begin{array}{c}\text { PER } \\
(\%)\end{array}$ & $\begin{array}{c}\text { PER Inc } \\
(\%)\end{array}$ \\
\hline Baseline & 23.8 & 0.0 & 22.8 & 0.0 & 22.7 & 0.0 & 20.8 & 0.0 \\
\hline \multicolumn{9}{|l|}{ Fill_0 } \\
\hline Landmark-keep & 36.1 & 51.7 & 33.4 & $46.5(1.34)^{* *}$ & 49.6 & 118.5 & 49.7 & $139(10.3)^{*}$ \\
\hline Random & 42.3 & 77.7 & 42.1 & $84.6(8.35)$ & 50.9 & 124.2 & 52.8 & $154(14.8)$ \\
\hline \multicolumn{9}{|l|}{ Copy } \\
\hline Landmark-keep & 35.2 & 47.7 & 32.3 & $41.5(1.08) * *$ & 29.4 & 29.3 & 26.9 & $29.3(0.653)^{* *}$ \\
\hline Random & 44.0 & 84.9 & 44.1 & $93.5(0.734)$ & 38.4 & 69.3 & 37.6 & $80.9(0.942)$ \\
\hline
\end{tabular}

For the next experiment we inverted the setup: instead of keeping only landmark frames, we drop only landmark frames (call this the Landmark-drop strategy). Table III compares the PER increment of a Landmark-drop strategy to the increment suffered by a Random frame 
drop strategy with the same percentage of lost frames. The Landmark-drop strategy always return higher PER. However, only for the GMM setup Copy did we obtain a significant $p$ value during cross validation. The $p$ values for other setups range from 0.13 to 0.17 . Again, the Wilcoxon test has been conducted on the default test set, with the conclusion that only the GMM Copy pair demonstrated significant difference.

TABLE III: PER increments for dropping Landmark frames during scoring compared to randomly dropping a similar portion of frames ( $C V$ stands for cross validation)

\begin{tabular}{|c|c|c|c|c|c|c|c|c|}
\hline Acoustic model & \multicolumn{4}{|c|}{ GMM } & \multicolumn{4}{|c|}{ DNN } \\
\hline Test regime & \multicolumn{2}{|c|}{ Default } & \multicolumn{2}{|c|}{ CV Mean (Stdev) } & \multicolumn{2}{|c|}{ Default } & \multicolumn{2}{|c|}{ CV Mean (Stdev) } \\
\hline Metric & $\begin{array}{c}\text { PER } \\
(\%)\end{array}$ & $\begin{array}{c}\text { PER Inc } \\
(\%)\end{array}$ & $\begin{array}{c}\text { PER } \\
(\%)\end{array}$ & $\begin{array}{c}\text { PER Inc } \\
(\%)\end{array}$ & $\begin{array}{c}\text { PER } \\
(\%)\end{array}$ & $\begin{array}{c}\text { PER Inc } \\
(\%)\end{array}$ & $\begin{array}{c}\text { PER } \\
(\%)\end{array}$ & $\begin{array}{c}\text { PER Inc } \\
(\%)\end{array}$ \\
\hline Baseline & 23.8 & 0.0 & 22.8 & 0.0 & 22.7 & 0.0 & 20.8 & 0.0 \\
\hline \multicolumn{9}{|l|}{ Fill_0 } \\
\hline Landmark-drop & 25.6 & 7.56 & 24.0 & $5.33(1.36)$ & 24.2 & 6.61 & 23.1 & $11.1(1.58)$ \\
\hline Random & 24.1 & 1.26 & 23.4 & $2.68(1.23)$ & 23.6 & 3.96 & 22.4 & $7.53(1.24)$ \\
\hline \multicolumn{9}{|l|}{ Copy } \\
\hline Landmark-drop & 25.6 & 7.5 & 24.1 & $5.83(0.873)^{*}$ & 24.3 & 7.1 & 22.1 & $6.44(0.836)$ \\
\hline Random & 24.6 & 3.3 & 23.1 & $1.14(0.948)$ & 23.6 & 4.0 & 21.6 & $3.85(0.760)$ \\
\hline
\end{tabular}

The results in Table II demonstrate that keeping landmark frames is better than keeping a random selection of frames at the same drop rate, in all but one of the tested comparison pairs. The results in Table III demonstrate that random selection tends to be better than selectively dropping the landmark frames, though the difference is only significant in one of the four comparison pairs. These two findings support the hypothesis that frames containing landmarks are more important than others. However, the PER increment in some setups are very large, indicating the ASR might no longer be functioning under stable conditions.

\section{Using Landmark as a Heuristic to Achieve Computation Reduction}

Methods in Table II and III compared the Landmark-keep, Landmark-drop, and Random frame drop strategies. Table IV] illustrates PER increment (\%) for the Landmark-keep and Regular frame-dropping strategies. In this experiment, we are no longer directly testing Hypothesis 2. Instead, we are trying to achieve high frame dropping rate subject to low PER increment. As dropped frames need not be calculated during the acoustic model scoring procedure, a high dropping ratio can benefit the ASR by reducing computational load. The strategy leveraging landmark information is a hybrid strategy: on top of a standard Regular 
strategy, it keeps all landmark frames and over-weights the likelihoods of these frames as in VIA. For each acoustic model type (GMM vs. DNN), three different percentage rates of frame dropping are exemplified. In each case, we select a Regular strategy with high dropping rate, modify it to keep the landmark frames, measure the percentage of frames dropped by the resulting strategy, then compare the result to a purely Regular frame-drop strategy with a similar drop rate. The baseline Regular strategies have three standard drop rates: $33.3 \%$ (one out of three frames dropped, uniformly), $50 \%$ (one out of two frames dropped), and $66.7 \%$ (two out of three frames dropped). Table IV highlights results for one of the setups in bold, as that setup achieves a very good trade off between high dropping ratio and low PER increment.

As we can see, for DNN acoustic models, the Landmark-keep strategy results in lower error rate increment than a Regular strategy dropping a similar number of frames. Wilcoxon tests demonstrated a statistically significant difference at all three drop rates. For GMM acoustic models, avoiding landmarks does not seem to return a lower error rate. In fact, the error rate is higher for 2 out of 3 different drop rates. The highlighted case in Table IV is intriguing because it the PER increment is so low, and this row will therefore serve as the basis for further experimentation in the next section. In this setup for DNN, over $50 \%$ of the frames were dropped, but the PER only increased by $0.44 \%$. This result seems to support the hypothesis that landmark frames contain more information for ASR than other frames, but in Table IV, this row has the appearance of an anomaly, since the error increment is so small. In order to confirm that this specific data point is not a special case, we conducted additional experiments with very similar setups. The results for these additional experiments are presented in Table $\mathrm{V}$.

Additional results presented in Table $\mathrm{V}$ are obtained through applying an over-weighting factor close to 4, which is the optimal value found for DNNs in Figure 3. The first and third rows in this table randomly keep or drop a small number of non-landmark frames, in order to obtain drop rates of $52.1 \%$ and $56.3 \%$ respectively. Since the selection is random, multiple runs of the experiment result in different PER for the same drop rate, therefore we repeated each experiment 10 times and reported the mathematical mean. Since there is a level of randomness in these results, we do not intend to evaluate our hypotheses on these data; rather, the goal of Table $\mathrm{V}$ is merely to confirm that the highlighted case in Table IV is a relatively stable result of its parameter settings, and not an anomaly. Since 
TABLE IV: PER increments comparison between Landmark-keep and Regular drop strategies for GMM and DNN.

\begin{tabular}{||c|r|r|r|r|r|r||}
\hline \multirow{2}{*}{ Copy } & \multicolumn{2}{|c|}{ Default } & \multicolumn{4}{|c||}{ Cross Validation } \\
\cline { 2 - 7 } & Drop Rate\% & PER Inc\% & Drop Rate\% & PER Inc\% & Inc STD\% & Inc pVal \\
\hline Land & 41.0 & 1.26 & 44.4 & 1.84 & 0.0133 & 0.962 \\
Reg & 33.3 & 3.78 & 33.3 & 1.81 & 0.0119 & \\
\hline Land & 54.2 & 2.94 & 54.1 & 2.86 & 0.0140 & 0.598 \\
Reg & 50 & 2.1 & 50 & 2.58 & 0.00780 & \\
\hline Land & 64.3 & 12.1 & 65.0 & 8.10 & 0.0182 & 0.159 \\
Reg & 66.7 & 10.1 & 66.7 & 6.91 & 0.0181 & \\
\hline Land & 41.0 & 0.44 & 44.4 & 1.84 & 0.0115 & 0.0011 \\
Reg & 33.3 & 3.98 & 33.3 & 4.20 & 0.0153 & \\
\hline Land & $\mathbf{5 4 . 2}$ & $\mathbf{0 . 4 4}$ & $\mathbf{5 8 . 4}$ & $\mathbf{1 . 9 0}$ & $\mathbf{0 . 1 6 7}$ & $\mathbf{0 . 0 0 2 9}$ \\
Reg & 50 & 2.21 & 50 & 4.12 & 0.0115 & \\
\hline Land & 64.2 & 3.08 & 69.0 & 5.86 & 0.0121 & 0.0391 \\
Reg & 66.7 & 6.17 & 66.7 & 7.04 & 0.0160 & \\
\hline
\end{tabular}

TABLE V: PER increments for Landmark-keeping strategy for DNN with dropping rate near $54.2 \%$ and over-weighting factor near 4 times

\begin{tabular}{||l|l|l|l|l||}
\hline PER Inc\% & & \multicolumn{4}{|c||}{ Over-weighting Factor } \\
\hline & & 3.5 & 4 & 4.5 \\
\hline \multirow{3}{*}{ Drop Rate\% } & 52.1 & 1.42 & 0.84 & 0.93 \\
\cline { 2 - 5 } & 54.2 & 0.88 & 0.44 & 0.88 \\
\cline { 2 - 5 } & 56.3 & 0.62 & 0.40 & 0.40 \\
\hline
\end{tabular}

good continuity can be observed across nearby settings, results in Table $\mathrm{V}$ lend support to the highlighted test case in Table IV.

\section{DISCUSSION}

Results in Section VIA tend to support hypothesis 1. However, the tendency is not statistically significant. The tendency is consistent for the GMM-based system, for all overweighting factors between 1.0 and 3.0. Similar tendencies appeared for over-weight factors between 3.0 and 5.0 for DNN-based system.

Experiments in Section VIB tested different non-landmark-based frame drop strategies, and different methods of frame replacement. It was shown that, among the several strategies tested, the Regular-Copy strategy obtains the smallest PER. There is an interesting synergy between the frame-drop strategy and the frame-replacement strategy, in that the PER of a $50 \%$ Regular-Copy system (one out of every two frames dropped) is even better than that of 
a 33\% Regular-Copy system (one out of every three frames dropped). This result, although surprising, confirms a similar finding reported by Sak et al. (2014). We suspect that the reason may be relevant to the regularity of the 50\% drop rate. When we drop 1 frame out of every 2 frames, the effective time span of each remaining frame is $20 \mathrm{~ms}$, with the frame extracted at the center of the time span. Dropping 1 frame out of every 3 frames, on the other hand, results in an effective time span per frame of $15 \mathrm{~ms}$, but the alignment of each frame's signal window to its assigned time span alternates from frame to frame.

It is worth mentioning that our definition of acoustic landmarks differs from that of Lulich (2010) - specifically, Lulich claims that there is no landmark in the center of Vowel and Glide. Instead, formant-subglottal resonance crossing, which is known to sit between the boundaries of [-Back] and [+Back] vowels, contains a landmark. It is possible that an alternative definition of landmarks might lead to better results.

We can also observe that GMM and DNN acoustic models tend to perform differently in the same setup. For example, for GMM, randomly dropping frames results in a higher PER than up-sampling; this is not the case for DNN models. Results also demonstrate that DNN models perform quite well when frames are missing. A PER increment of only $6 \%$ occurs after throwing away $2 / 3$ of the frames. GMM models tend to do much worse, especially when the drop rate goes up.

All experiments on DNN tend to support the strategy to avoid dropping landmarks. However, the 2 test cases covered in Table III lack statistical confidence. Scoring only the landmark frames (the Landmark-keep strategy) out-performs both Random and Regular framedrop-strategies. On the other hand, if landmark frames are dropped (the Landmark-drop strategy), we obtain higher PER when compared to randomly scoring a similar number of frames.

We find, at least for ASR with DNN acoustic models, that landmark frames contain information that is more useful to ASR than other frames. In the most striking case, the highlighted result in Table IV indicates that it is possible to drop more than $54 \%$ of the frames but only observe a $0.44 \%$ increment in the PER compared to baseline (PER increases from 22.7 to 22.8). We conclude, for DNN-based ASR, that experiments support hypothesis 2 (with statistically significant differences in two out of the three comparisons). In comparison, we failed to find support for hypothesis 2 in GMM-based ASR. 


\section{A. How Landmarks Affect the Decoding Results}

Having proven that the Landmark-keep strategy is more effective than a Random or Regular drop strategy, we proceeded to investigate the resulting changes in the rates of insertion, deletion and confusion among phones. We compared the normalized increment of each type of error, separately, when the confusion matrices of the baseline system are subtracted from the confusion matrices of the Landmark-keep and Random frame-drop systems. Fig. 6 compares the normalized error increment, of different types of errors, for the Landmark-keep and Random strategies. The numbers reported in the figure are normalized error increment. They are calculated using error increment divided by the occurrence of each kind of phone. We use this measure to reflect the increment ratio while avoiding having to deal with situations that could lead to division by zero.

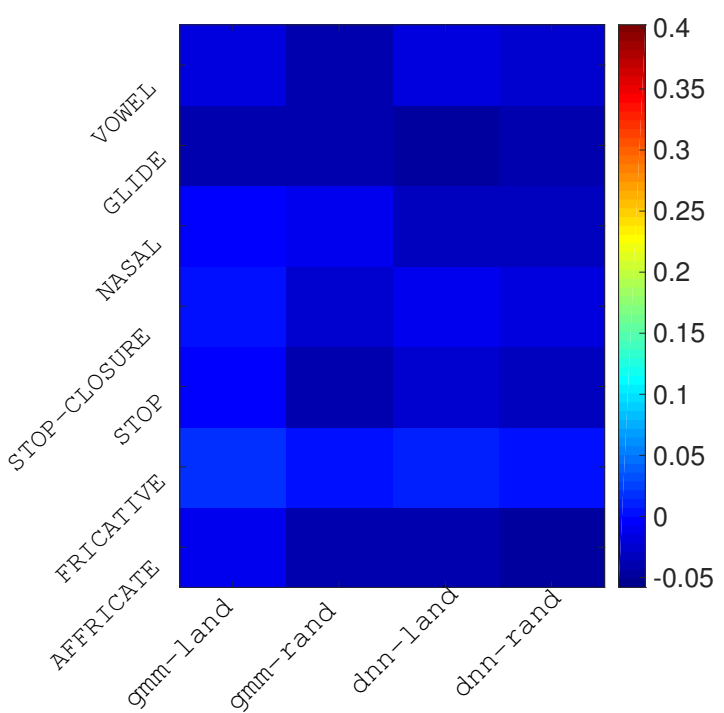

(a) Insertion errors

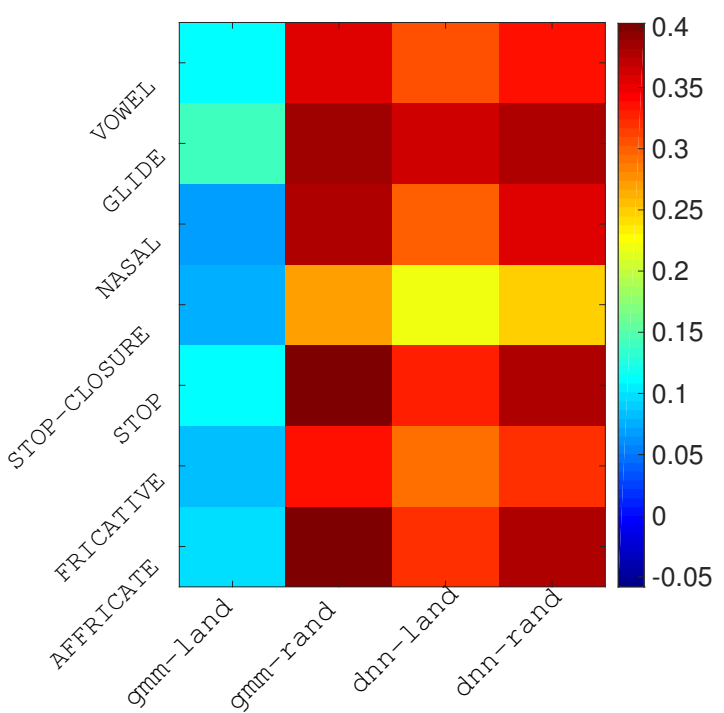

(b) Deletion errors

FIG. 6: The normalized error increment for a) insertion errors and b) deletion errors (y-axis represent different manners of articulators and $x$-axis represent different systems)

Overall, dropping frames causes a minor reduction to the phone insertion rate, while the phone deletion rate significantly worsens. We suspect that after dropping frames, the decoder is less effective at capturing transitions between phones, resulting in correctly detected phones spanning over other phones. In Figure $6 \mathrm{~b}$ we can see that the Landmark-keep 
strategy is more effective than the Random strategy, since it returns a lower deletion rate increment. We believe this is because the landmark contains sufficient acoustic information about each phone to force it to be recognized. However, we do not know why the GMM-Landmark-keep strategy is less effective at preventing phone deletions than the DNNLandmark-keep strategy. A possible reason might be that more frames were stacked together in the splicing process for the DNN than for the GMM (Veselý et al., 2013) If we do consider providing landmarks as extra information to ASR, in order to reduce computation load for example, the difference between GMM and DNN models should be considered.

\section{CONCLUSIONS}

Phones can be categorized using binary distinctive features, which can be extracted through acoustic cues anchored at acoustic landmarks in the speech utterance. In this work, we proved through experiments for DNN-based ASR systems operating on MFCC features, on the TIMIT corpus, using both the default and cross validation train-test splits, that frames containing landmarks are more informative than others. We proved that paying extra attention to these frames can potentially compensate for accuracy lost when dropping frames during Acoustic Model likelihood scoring. We leveraged the help of landmarks as a heuristic to guide frame dropping during speech recognition. In one setup, we dropped more than $54 \%$ of the frames while adding only $0.44 \%$ to the Phone Error Rate. This demonstrates the potential of landmarks for computational reduction for ASR systems with DNN acoustic models. We conclude that a DNN-based system is able to find a nearly-sufficient summary of the entire spectrogram in frames containing acoustic landmarks, in the sense that, if computational considerations require one to drop $50 \%$ or more of all speech frames, one is better off keeping the landmark frames than keeping any other tested set of frames. GMM-based experiments return mixed results, but results for the DNN are consistent and statistically significant: landmark frames contain more information about the phone string than frames without landmarks. 
Borys, S. (2008), An SVM front-end landmark speech recognition system, Master's thesis (University of Illinois at Urbana-Champaign, Urbana, IL, USA).

Chitturi, R., and Hasegawa-Johnson, M. (2006), "Novel time domain multi-class svms for landmark detection." in Proceedings of Annual Conference of the International Speech Communication Association (INTERSPEECH) (International Speech and Communication Association (ISCA), Baixas France) pp. 2354-2357.

Choi, J.-Y. (1999), "Detection of consonant voicing: A module for a hierarchical speech recognition system," in The Journal of the Acoustical Society of America, Vol. 106 (Acoustical Society of America, Melville NY USA) p. 2274.

Chomsky, N., and Halle, M. (1968), "The sound pattern of english," (MIT Press, Cambridge MA USA) pp. 1-484.

Cressie, N., and Whitford, H. (1986), "How to use the two sample t-Test," in Biometrical Journal, Vol. 28 (Wiley Online Library) pp. 131-148.

Fletcher, H., and Munson, W. A. (1933), "Loudness, its definition, measurement and calculation," in Bell System Technical Journal, Vol. 12 (Wiley Online Library) pp. 377-430.

Furui, S. (1981), "Cepstral analysis technique for automatic speaker verification," in IEEE Transactions on Acoustics, Speech, and Signal Processing, Vol. 29 (Institute of Electrical and Electronics Engineers (IEEE), Piscataway New Jersey US) pp. 254-272.

Furui, S. (1986), "On the role of spectral transition for speech perception," in The Journal of the Acoustical Society of America, Vol. 80 (Acoustical Society of America, Melville NY USA) pp. $1016-1025$.

Garofalo, J. S., Lamel, L. F., Fisher, W. M., Fiscus, J. G., Pallett, D. S., and Dahlgren, N. L. (1993), "The DARPA TIMIT acoustic-phonetic continuous speech corpus cdrom," in Linguistic Data Consortium.

Gillick, L., and Cox, S. J. (1989), "Some statistical issues in the comparison of speech recognition algorithms," in Proceedings of IEEE International Conference on Acoustics, Speech, and Signal Processing (ICASSP) (Institute of Electrical and Electronics Engineers (IEEE), Piscataway New Jersey US) pp. 532-535.

Hasegawa-Johnson, M. (2000), "Time-frequency distribution of partial phonetic information mea- 
sured using mutual information," in Proceedings of 6th International Conference on Spoken Language Processing (ICSLP) (China Military Friendship Publish, China) pp. 133-136.

Hasegawa-Johnson, M., Baker, J., Borys, S., Chen, K., Coogan, E., Greenberg, S., Juneja, A., Kirchhoff, K., Livescu, K., Mohan, S., et al. (2005), "Landmark-based speech recognition: Report of the 2004 Johns Hopkins summer workshop," in Proceedings of IEEE International Conference on Acoustics, Speech, and Signal Processing (ICASSP), Vol. 1 (Institute of Electrical and Electronics Engineers (IEEE), Piscataway New Jersey US) p. 1213.

Hermansky, H. (2003), "Trap-tandem: Data-driven extraction of temporal features from speech," in Automatic Speech Recognition and Understanding, 2003. ASRU'03. 2003 IEEE Workshop on (Institute of Electrical and Electronics Engineers (IEEE)) pp. 255-260.

Howitt, A. W. (2000), "Vowel landmark detection," in Proceedings of Annual Conference of the International Speech Communication Association (INTERSPEECH) (International Speech and Communication Association (ISCA), Baixas France) pp. 628-631.

Iso-Sipilä, J. (2000), "Speech recognition complexity reduction using decimation of cepstral time trajectories," in Proceedings of 10th IEEE European Signal Processing Conference (Institute of Electrical and Electronics Engineers (IEEE), Piscataway New Jersey US) pp. 1-4.

Jakobson, R., Fant, C. G., and Halle, M. (1951), "Preliminaries to speech analysis. the distinctive features and their correlates." (MIT Press, Cambridge MA USA) pp. 1-64.

Jansen, A., and Niyogi, P. (2008), "A hierarchical point process model for speech recognition," in Proceedings of IEEE International Conference on Acoustics, Speech and Signal Processing (ICASSP) (Institute of Electrical and Electronics Engineers (IEEE), Piscataway New Jersey US) pp. 4093-4096.

Juneja, A. (2004), Speech recognition based on phonetic features and acoustic landmarks, Ph.D. thesis (University of Maryland at College Park, College Park, MD, USA).

Kirchhoff, K. (1998), "Combining articulatory and acoustic informatino for speech recognition in noisy and reverberant environments," in Proceedings of International Conference on Spoken Language Processing (ICSLP) (International Speech and Communication Association (ISCA), Baixas France) pp. 0873:1-4.

Kirchhoff, K. (1999), Robust speech recognition using articulatory information, Ph.D. thesis (University of Bielefeld).

Kirchhoff, K., Finkard, G. A., and Sagerer, G. (2002), "Combining acoustic and articulatory feature 
information for robust speech recognition," in Speech Communication, Vol. 37, pp. 303-319.

Kong, X., Yang, X., Hasegawa-Johnson, M., Choi, J.-Y., and Shattuck-Hufnagel, S. (2016), "Landmark-based consonant voicing detection on multilingual corpora," in arXiv preprint arXiv:1611.03533.

Lee, C.-H., Clements, M. A., Dusan, S., Fosler-Lussier, E., Johnson, K., Juang, B.-H., and Rabiner, L. R. (2007), "An overview on automatic speech attribute transcription (asat)." in Proceedings of Annual Conference of the International Speech Communication Association (INTERSPEECH) (International Speech and Communication Association (ISCA), Baixas France) pp. 1825-1828.

Lee, J.-J., and Choi, J.-Y. (2008a), "Detection of obstruent consonant landmark for knowledge based speech recognition system," in Acoustics '08 (Paris) pp. 2417-2421.

Lee, J.-W., Choi, J.-Y., and Kang, H.-G. (2011), "Classification of fricatives using feature extrapolation of acoustic-phonetic features in telephone speech," in Proceedings of Annual Conference of the International Speech Communication Association (INTERSPEECH) (International Speech and Communication Association (ISCA), Baixas France) pp. 1261-1264.

Lee, J.-W., Choi, J.-Y., and Kang, H.-G. (2012), "Classification of stop place in consonant-vowel contexts using feature extrapolation of acoustic-phonetic features in telephone speech," in The Journal of the Acoustical Society of America, Vol. 131 (Acoustical Society of America, Melville NY USA) pp. 1536-1546.

Lee, K.-F. (1988), "Automatic speech recognition: the development of the sphinx system," (Springer Science \& Business Media, Berlin Germany) pp. 1-207.

Lee, S., and Choi, J.-Y. (2008b), "Vowel place detection for a knowledge-based speech recognition system," in Acoustics '08 (Paris) pp. 2430-2433.

Liu, S. A. (1996), "Landmark detection for distinctive feature-based speech recognition," in The Journal of the Acoustical Society of America, Vol. 100 (Acoustical Society of America, Melville NY USA) pp. 3417-3430.

Livescu, K., Çetin, Özgür., Hasegawa-Johnson, M., King, S., Bartels, C., Borges, N., Kantor, A., Lal, P., Yung, L., Bezman, A., Dawson-Hagerty, S., Woods, B., Frankel, J., Magimai-Doss, M., and Saenko, K. (2007), "Articulatory feature-based methods for acoustic and audio-visual speech recognition: 2006 JHU summer workshop final report," in Proceedings of IEEE International Conference on Acoustic, Speech, and Signal Processing (ICASSP) (Institute of Electrical and Electronics Engineers (IEEE), Piscataway New Jersey US) pp. 621-624. 
Lulich, S. M. (2010), "Subglottal resonances and distinctive features," in Journal of Phonetics, Vol. 38 (Elsevier, Amsterdam Netherlands) pp. 20-32.

McGraw, I., Prabhavalkar, R., Alvarez, R., Arenas, M. G., Rao, K., Rybach, D., Alsharif, O., Sak, H., Gruenstein, A., Beaufays, F., et al. (2016), "Personalized speech recognition on mobile devices," in Proceedings of International Conference on Acoustics, Speech and Signal Processing (ICASSP) (Institute of Electrical and Electronics Engineers (IEEE), Piscataway New Jersey US) pp. $5955-5959$.

Metze, F. (2005), Articulatory Features for Conversational Speech Recognition, Ph.D. thesis (Fakultät für Informatik der Universität Karlsruhe (TH), Karlsruhe, Germany).

Naess, A. B., Livescu, K., and Prabhavalkar, R. (2011), "Articulatory feature classification using nearest neighbors," in Proceedings of Annual Conference of the International Speech Communication Association (INTERSPEECH) (International Speech and Communication Association (ISCA), Baixas France) pp. 2301-2304.

Niyogi, P., Burges, C., and Ramesh, P. (1999), "Distinctive feature detection using support vector machines," in Proceedings of IEEE International Conference on Acoustics, Speech, and Signal Processing (ICASSP) (Institute of Electrical and Electronics Engineers (IEEE), Piscataway New Jersey US) pp. 425-428.

Ohde, R. N. (1994), "The developmental role of acoustic boundaries in speech perception," in The Journal of the Acoustical Society of America, Vol. 96 (Acoustical Society of America, Melville NY USA) pp. 3307-3307.

Öhman, S. E. G. (1965), "Coarticulation in vcv utterances: Spectrographic measurements," in The Journal of the Acoustical Society of America, Vol. 37 (Acoustical Society of America, Melville NY USA) pp. 151-168.

Qian, K., Zhang, Y., and Hasegawa-Johnson, M. (2016), "Application of local binary patterns for SVM based stop consonant detection," in Proc. Speech Prosody (International Speech and Communication Association (ISCA), Baixas France) pp. 1114-1118.

Quatieri, T. F. (2008), "Discrete-time speech signal processing: Principles and practice," (Pearson Education, Upper Saddle River NJ USA) pp. 1-816.

Sak, H., Senior, A., Rao, K., and Beaufays, F. (2015), "Fast and accurate recurrent neural network acoustic models for speech recognition," in arXiv preprint arXiv:150\%.06947.

Sak, H., Senior, A. W., and Beaufays, F. (2014), "Long short-term memory recurrent neural 
network architectures for large scale acoustic modeling." in Proceedings of 15th Annual Conference of the International Speech Communication Association (INTERSPEECH) (International Speech and Communication Association (ISCA), Baixas France) pp. 338-342.

STEVENS, K. (1972), "The quantal nature of speech: Evidence from articulatory-acoustic data," in Human Communication: A Unified View (McGraw Hill).

Stevens, K. N. (1985), "Evidence for the role of acoustic boundaries in the perception of speech sounds," in Phonetic Linguistics: Essays in Honor of Peter Ladefoged, edited by V. A. Fromkin (Academic Press, Cambridge MA USA, Orlando, Florida) pp. 243-255.

Stevens, K. N. (2000), "Acoustic phonetics," (MIT Press, Cambridge MA USA, Cambridge) pp. 1-615.

Stevens, K. N. (2002), "Toward a model for lexical access based on acoustic landmarks and distinctive features," in The Journal of the Acoustical Society of America, Vol. 111 (Acoustical Society of America, Melville NY USA) pp. 1872-1891.

Stevens, K. N., Keyser, S. J., and Kawasaki, H. (1986), "Toward a phonetic and phonological theory of redundant features," in Invariance and Variability in Speech Processes, edited by J. S. Perkell and D. H. Klatt (Lawrence Erlbaum Associates, New Jersey USA, Hillsdale, NJ US) pp. 426-463.

Stevens, K. N., Manuel, S. Y., Shattuck-Hufnagel, S., and Liu, S. (1992), "Implementation of a model for lexical access based on features," in Proceedings of International Conference on Spoken Language Processing (ICSLP), Vol. 1 (International Speech and Communication Association (ISCA), Baixas France, Banff, Alberta) pp. 499-502.

Vanhoucke, V., Devin, M., and Heigold, G. (2013), "Multiframe deep neural networks for acoustic modeling," in Proceedings of IEEE International Conference on Acoustics, Speech and Signal Processing (ICASSP) (Institute of Electrical and Electronics Engineers (IEEE), Piscataway New Jersey US) pp. 7582-7585.

Veselý, K., Ghoshal, A., Burget, L., and Povey, D. (2013), "Sequence-discriminative training of deep neural networks," in Proceedings of Annual Conference of the International Speech Communication Association (INTERSPEECH) (International Speech and Communication Association (ISCA), Baixas France) pp. 2345-2349.

Wang, S., Lulich, S. M., and Alwan, A. (2009), "Automatic detection of the second subglottal resonance and its application to speaker normalization a," in The Journal of the Acoustical Society 
of America, Vol. 126 (Acoustical Society of America, Melville NY USA) pp. 3268-3277.

Xie, Z., and Niyogi, P. (2006), "Robust acoustic-based syllable detection." in Proceedings of Annual Conference of the International Speech Communication Association (INTERSPEECH) (International Speech and Communication Association (ISCA), Baixas France) p. 1327.

Xiong, W., Droppo, J., Huang, X., Seide, F., Seltzer, M., Stolcke, A., Yu, D., and Zweig, G. (2016), "Achieving human parity in conversational speech recognition," in arXiv preprint arXiv:1610.05256. 